\title{
Spatially Resolved X-ray Fluorescence Analysis with a Pyroelectric X-ray Emitter
}

\author{
So Hatakeyama, Shinsuke Kunimura, Nobuharu SaSaki, Takashi Yamamoto, ${ }^{\dagger}$ and Jun Kawai \\ Department of Materials Science and Engineering, Kyoto University, Kyoto 606-8501, Japan
}

\begin{abstract}
A portable instrument for two-dimensional X-ray fluorescence imaging was assembled with an X-ray source using a pyroelectric crystal, which was driven by a 9-V dry battery, a Si-PIN detector, a slit, and pulse motors. Line scanning for a mug and a knife-edge-scan of an iron sheet were carried out using this spectrometer. The sensitivity of the spectrometer was sufficient for elemental analysis of a mug using a $1 \mathrm{~mm}^{2}$ slit, and several elements, such as $\mathrm{Co}, \mathrm{Ni}, \mathrm{Zn}, \mathrm{Pb}$ and $\mathrm{Zr}$, were detected. The estimated spatial resolution using a $0.8-\mathrm{mm}$ pinhole was $3.5 \mathrm{~mm}$.
\end{abstract}

(Received April 29, 2008; Accepted May 13, 2008; Published July 10, 2008)

\section{Introduction}

The development of a portable X-ray fluorescence spectrometer has been the subject of intensive study for on-site analysis for various samples, such as environmental samples, articles for sale, works of art, and archaeological artifacts. The analysis of micro areas has also been required. A pyroelectric-type X-ray emitter developed by Brownridge ${ }^{1,2}$ is one of the prime candidates for the $\mathrm{X}$-ray source of a portable analyzer. Brownridge and his colleague reported that changing the temperature of a pyroelectric crystal, such as $\mathrm{CsNO}_{3},{ }^{1} \mathrm{LiTaO}_{3}{ }^{2}$ or $\mathrm{LiNbO}_{3},{ }^{2}$ at appropriate pressures caused charging on the surface, and that X-rays were emitted by the impact of electrons alternately on a metal target and the charged surface along with the Z-axis. A miniature X-ray emitter, "Cool-X", with a pyroelectric crystal has been commercially available, which was developed based on their studies. The dimensions are as small as $15 \mathrm{~mm}$ in diameter and $10 \mathrm{~mm}$ in height. When it is installed in a controller box with a 9-V dry battery, which is utilized for the temperature control of a pyroelectric crystal, dimensions of the device are $125 \mathrm{~mm}$ in length, $45 \mathrm{~mm}$ in width, and $30 \mathrm{~mm}$ in height. The pyroelectric X-ray emitter is thus useful for a portable XRF spectrometer because of its small dimensions and low-power.

Our research group assembled this commercially available pyroelectric X-ray emitter and a Si-PIN detector, and applied it to the X-ray fluorescence spectrometer in $2004 .^{3}$ They have subsequently been utilized for the identification of paint and leather, ${ }^{4}$ glass and ceramics, ${ }^{5}$ steel, ${ }^{6}$ daily necessaries, such as printing paper, business cards, cinnabar seal inks, aluminum foils and wrapping films. ${ }^{3,7,8}$ Environmental reference samples, ${ }^{9}$ rice and rice bran ${ }^{10}$ were also analyzed. By using of an X-ray fluorescence setup with a pyroelectric X-ray emitter and a diskshaped secondary target, Ida and Kawai succeeded in the quantitative analysis of $\mathrm{Cr}$ in steel (\% level). ${ }^{11}$ Trace quantitative analysis and detection at less than $1 \mathrm{mg} \mathrm{l}^{-1}$ were achieved using

† To whom correspondence should be addressed.

E-mail: t.yamamoto@materials.mbox.media.kyoto-u.ac.jp a simple preconcentration procedure. ${ }^{12}$ Except for our research group, however, the application of this X-ray source has been scarcely reported.

As the next step, we planned to apply this pyroelectric X-ray emitter to an X-ray source for a portable two-dimensional elemental analyzer. Although a portable X-ray fluorescence spectrometer accompanied by spatial resolution capability has been reported by several research groups, ${ }^{13-18}$ the X-ray sources utilized were conventional X-ray tubes. In this study, we assembled an X-ray fluorescence spectrometer with a pyroelectric X-ray emitter, a Si-PIN X-ray detector, a slit and two position-controlled sample stages; elemental mapping of a mug and a knife-edge-scan of an iron sheet were carried out to assess the capability of this X-ray emitter for two-dimensional $\mathrm{X}$-ray fluorescence analysis.

\section{Experimental}

\section{Instrument}

A two-dimensional X-ray fluorescence spectrometer was assembled with a pyroelectric X-ray source Cool-X (Amptek Inc., Bedford, MA), a Si-PIN detector, a slit made of Teflon or $\mathrm{Cu}$ sheet, a translation motorized stage, and a rotation motorized stage. Figure 1 shows a photograph of the instrument, and the geometry is illustrated in Fig. 2. The translation and rotation motorized pulse stage utilized were LTS-200 (Sigma Koki Co. Ltd., Japan) and MINI-600z (Sigma Koki Co. Ltd.), respectively. The sample positions were computationally controlled by software programmed with LabVIEW (National Instruments Co., Austin, TX). Fluorescent X-rays were detected by using a Si-PIN detector XR-100CR (Amptek) containing a preamplifier. Voltage signals from the pre-amplifier were amplified by using an amplifier PX2CR (Amptek) and the amplified signals were taken into a Microsoft Windows computer through a multi-channel analyzer (MCA) MCA8000A (Amptek). The number of channels of the MCA was set to 512, and one channel corresponded to an X-ray energy of $41 \mathrm{eV}$ at the setting.

The details of the X-ray source utilized were reported in a previous paper, ${ }^{8,19}$ and are shortly summarized as follows: (1) 


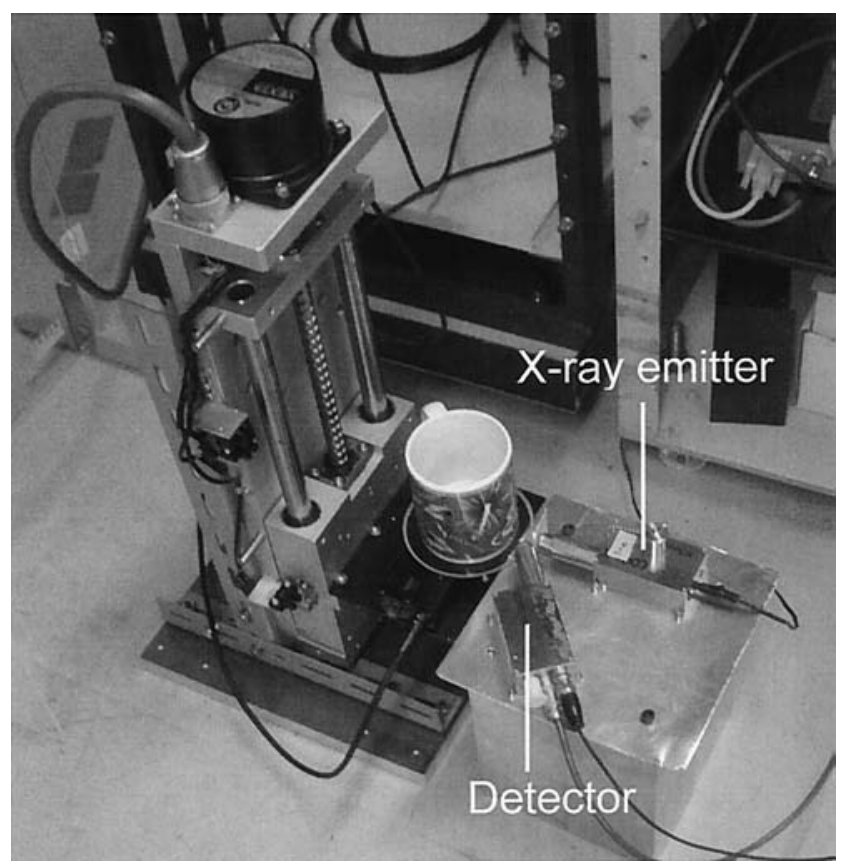

Fig. 1 Schematic view of the instrument.

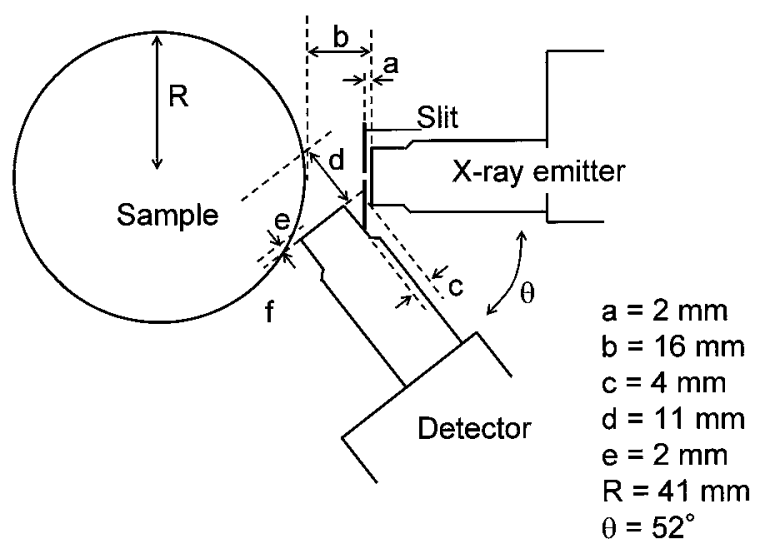

Fig. 2 Geometry among the X-ray emitter, the detector, the slit and the sample.

The X-ray source mainly consists of $\mathrm{LiTaO}_{3}$ as a pyroelectric crystal and $\mathrm{Cu}$ as a target material, (2) the surface of the crystal becomes positively or negatively charged when it is heated or cooled, (3) X-rays are emitted by the cyclic charging, and (4) the X-ray emitted are $\mathrm{Cu}$ and $\mathrm{Ta}$ characteristic X-rays and continuous X-rays up to $35 \mathrm{kV}$. According to Amptek, Inc., ${ }^{20}$ the electric power for X-ray production is less than $300 \mathrm{~mW}$, and is supplied by a $9-\mathrm{V}$ dry battery. The $\mathrm{X}$-ray output flux varies throughout the heating-cooling cycle $(\sim 2 \mathrm{~min})$ and the maximum is less than $10^{8}$ photons/s.

\section{Measurements}

The mug shown in Fig. 3 and an iron sheet were utilized for the present study. The surface of the mug was painted green, blue, red, or white. A Teflon ${ }^{\circledR}$ (poly-tetra-fluoroethylene, PTFE) sheet $(0.5 \mathrm{~mm}$ thickness $)$ with a $1-\mathrm{mm}^{2}$ aperture was placed between the X-ray source and the mug in order to restrict the size of the primary X-rays. An iron sheet with a thickness of 1.2 $\mathrm{mm}$ was measured in order to estimate the spatial resolution of

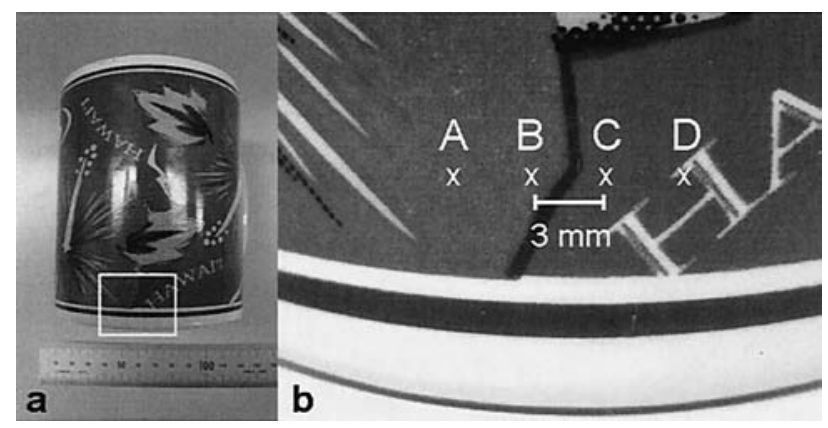

Fig. 3 Measured mug (a) and four measured points (b). A, B, Red; $\mathrm{C}, \mathrm{D}$, blue areas.

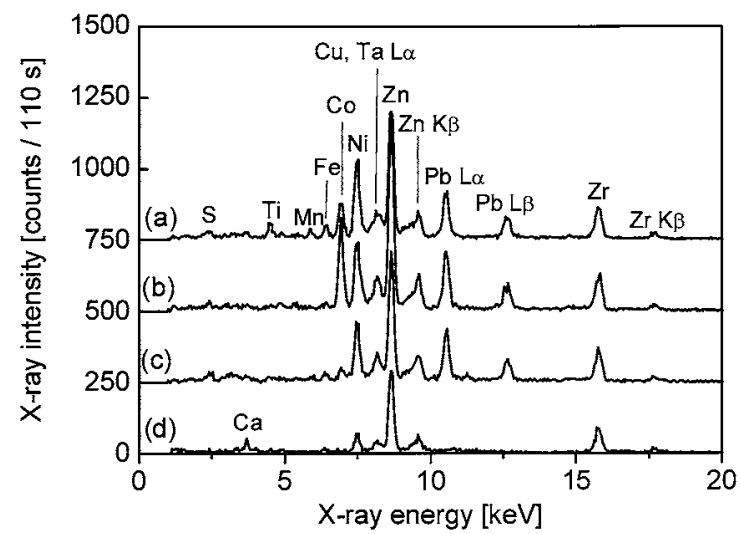

Fig. 4 XRF spectra of the mug. (a) Green, (b) blue, (c) red, (d) white sections.

the instrument. In measurements of the iron sheet, a $\mathrm{Cu}$ sheet (1.5 mm thickness) with a pin hole $(0.8 \mathrm{~mm}$ in diameter) was placed in front of the X-ray source. The measurements at each point were performed in air for 110 or $40 \mathrm{~s}$. As described above, the X-ray output flux varies during the thermal cycle. The incident X-rays for the present 40 and $110 \mathrm{~s}$ measurements are for a single heating phase and a single heating-cooling phase, respectively. Prior to a measurement, the X-ray emitter had been switched off for at least $5 \mathrm{~min}$ to be sure that each total flux of the emitted X-ray was constant.

\section{Results and Discussion}

Figure 4 shows the XRF spectra of the mug measured in (a) green, (b) blue, (c), red, and (d) white sections. The $\mathrm{Cu} K$ and Ta $L$ lines were due to the characteristic X-rays from the X-ray source. Emission lines due to the $\mathrm{Zn}$ and $\mathrm{Zr}$ species were detected at all of the spots. The $\mathrm{S}, \mathrm{Fe}, \mathrm{Co}, \mathrm{Ni}$, and $\mathrm{Pb}$ were detected at the red, blue and green areas. Most kinds of elements were detected in the green section; i.e., emission lines for $\mathrm{Ti}$ and $\mathrm{Mg}$ were also detected accompanied by those for $\mathrm{Co}$, $\mathrm{Ni}, \mathrm{Zn}, \mathrm{Pb}$ and $\mathrm{Zr}$. It should be noted that the net intensity of the Co $K_{\alpha}$ line was the highest at the blue area, while it was negligible at the red area. This clearly shows that the sensitivity of the present spectrometer using a $1-\mathrm{mm}^{2}$ slit is sufficient to distinguish the elemental composition, and that the Co $K_{\alpha}$ line is useful to assess the spatial resolution.

Then, X-ray fluorescence spectra of the mug around the boundary between red and blue areas were recorded in $3 \mathrm{~mm}$ 


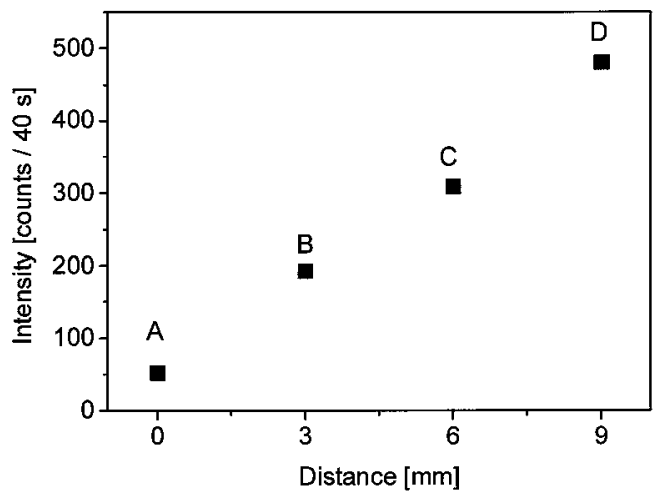

Fig. 5 Relationship between the measured points and the net intensities of the Co $K_{\alpha}$ peak.

intervals; the measuring position is indicated in Fig. 3. Positions $(\mathrm{A}, \mathrm{B})$ are red and $(\mathrm{C}, \mathrm{D})$ are blue. The net intensities of the $\mathrm{Pb}$ $L$ and $\mathrm{Zr} K$ lines did not change systematically, and were almost constant within $15 \%$ of the relative standard deviations. Figure 5 shows the net intensities of the Co $K_{\alpha}$ line for each position. In contrast to our assumption, the stepwise change of the net intensity was not confirmed when the measuring position varied from position $\mathrm{A}$ through $\mathrm{D}$, but the intensity increased monotonously. This result indicates that the spatial resolution of this setting was less than the size of the Teflon slit $(1 \times 1 \mathrm{~mm}$, $0.5 \mathrm{~mm}$ thickness), because of three possible reasons: (1) the transmission of the Teflon sheet utilized had a nonzero value of 0.24 at the energy for the $\mathrm{Cu} K_{\alpha}$ line, which was calculated using the composition of Teflon, the density, and the massabsorption coefficients ${ }^{21}$ obtained by Victoreen's coefficients; (2) the blue area on the mug was overcoated with the red area; (3) the size of X-ray beam at the sample position was larger than $1 \mathrm{~mm}^{2}$.

To evaluate the spatial resolution in more detail, a knife-edgescan of an iron sheet was finally performed along with the horizontal direction. This measurement was carried out with the same geometry as shown in Fig. 2, and an iron sheet was placed perpendicular to the X-ray emitter. A Cu sheet $(1.5 \mathrm{~mm}$ of thickness) with a hole of $0.8 \mathrm{~mm}$ diameter was placed in front of the X-ray emitter. The $\mathrm{Cu}$ sheet was selected because the transmittance for X-rays below $20 \mathrm{keV}$ is less than 0.01 , and $\mathrm{Cu}$ $K_{\alpha}$ line does not interfere with $\mathrm{Fe} K_{\alpha}$ line if it is emitted. Note that an incident X-ray, itself, contains the characteristic X-ray of $8.1 \mathrm{keV}$ due to $\mathrm{Cu} K_{\alpha}$ and/or Ta $L_{\alpha}$ lines. Thus, $\mathrm{Cu}$ is the best element for a slit. Figure 6 shows the measured net intensity of the Fe $K_{\alpha}$ peak and the result of a fitting by a sigmoidal curve. The first derivative of the fitted curve is also shown, and the evaluated spatial resolution obtained by the full width at half maximum of the curve was $3.5 \mathrm{~mm}$.

The preset X-ray emitter is driven by a dry battery that is used for the temperature control of an equipped pyroelectric crystal. Therefore, the all dimensions of the X-ray source and the weight are much less than those of a conventional X-ray tube. Regardless of the lower output power, the sensitivity for the present elemental analysis was sufficient, even when using a small $1 \mathrm{~mm}^{2}$ slit, and a spatial resolution of mm level was achieved. The output angle for 50\% intensity of the X-ray generator Cool- $\mathrm{X}$ is $140^{\circ} .20$ Thus, the spread of an incident $\mathrm{X}$ ray beam suppressed the spatial resolution as shown in Fig. 5. Because a pin hole was simply utilized in order to restrict the incident X-rays in the present study, the spatial resolution would be improved by using an appropriate slit. In conclusion, the

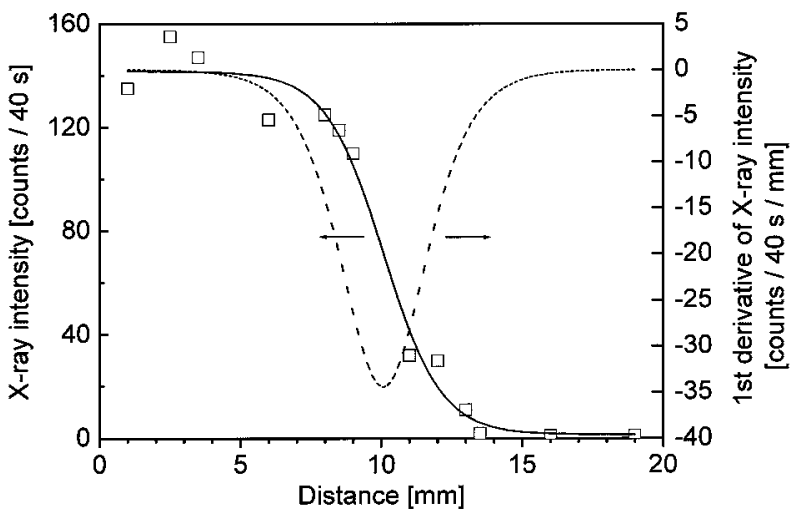

Fig. 6 X-ray intensity of an Fe sheet and the 1st derivative of the $\mathrm{X}$-ray intensity.

assembled X-ray fluorescence spectrometer using a pyroelectric $\mathrm{X}$-ray source has a potential to be used as a portable X-ray imaging analyzer.

\section{Conclusions}

A portable analyzer for two dimensional X-ray fluorescence imaging was assembled with an $\mathrm{X}$-ray source using a pyroelectric crystal, a Si-PIN detector, a slit and pulse motors. A line scan for a mug and a knife-edge-scan of an iron experiments were carried out. The $\mathrm{S}, \mathrm{Ca}, \mathrm{Ti}, \mathrm{Mn}, \mathrm{Fe}, \mathrm{Co}, \mathrm{Ni}$, $\mathrm{Zn}, \mathrm{Pb}$ and $\mathrm{Zr}$ were detected from the mug for $110 \mathrm{~s}$ measurements using a $1-\mathrm{mm}^{2}$ slit. Different kinds of elements were detected according to each color on the surface, and the Co $K_{\alpha}$ line was detected significantly at the blue section. The line scan of a mug with a Teflon slit did not give satisfactory spatial resolution of elemental imaging due to spreading of the incident X-ray beam and insufficient absorption coefficient of the Teflon sheet. The evaluated spatial resolution for this apparatus using an Fe sheet and a pin hole made of $\mathrm{Cu}$ was $3.5 \mathrm{~mm}$.

\section{Acknowledgements}

This work was supported by the Murata Science Foundation.

\section{References}

1. J. D. Brownridge, Nature, 1992, 358, 287.

2. J. D. Brownridge and S. Raboy, J. Appl. Phys., 1999, 86, 640.

3. J. Kawai, T. Yamada, and H. Fujimura, Bunseki Kagaku, 2004, 53, 183.

4. H. Ida and J. Kawai, Bunseki Kagaku, 2004, 53, 753.

5. H. Ida and J. Kawai, Anal. Sci., 2004, 20, 1211.

6. H. Ida and J. Kawai, Forensic Sci. Int., 2005, 151, 267.

7. H. Ida and J. Kawai, Adv. X-ray Chem. Anal. Jpn. (X-sen Bunseki no Shinpo, in Japanese), 2004, 35, 155.

8. H. Ida and J. Kawai, X-Ray Spectrom., 2005, 34, 225.

9. H. Ishii, H. Miyauchi, T. Hioki, and J. Kawai, Adv. X-ray Chem. Anal. Jpn. (X-sen Bunseki no Shinpo, in Japanese), 2005, 36, 225.

10. H. Ishii, N. Sato, M. Kawano, T. Mesaki, and J. Kawai, Bunseki Kagaku, 2005, 54, 321. 
11. H. Ida and J. Kawai, Spectrochim. Acta, Part B, 2005, 60, 89.

12. H. Ida and J. Kawai, J. Anal. At. Spectrom., 2004, 19, 1524.

13. P. Moioli and C. Seccaroni, X-Ray Spectrom., 2000, 29, 48.

14. M. Uda, Nucl. Instrum. Methods Phys. Res., Sect. B, 2004, 226,75 .

15. C. Zarkadas and A. G. Karydas, Spectrochim. Acta, Part B, 2004, 59, 1611.

16. M. Uda, A. Ishizaki, R. Satoh, K. Okada, Y. Nakajima, D. Yamashita, K. Ohashi, Y. Sakuraba, A. Shimono, and D. Kojima, Nucl. Instrum. Methods Phys. Res., Sect. B, 2005,
$239,77$.

17. I. Nakai, S. Yamada, Y. Terada, Y. Shindo, and T. Utaka, $X$-Ray Spectrom., 2005, 34, 46.

18. D. N. Papadopoulou, G. A. Zachariadis, A. N. Anthemidis, N. C. Tsirliganis, and J. A. Stratis, Talanta, 2006, 68, 1692.

19. H. Ida and J. Kawai, Adv. X-ray Chem. Anal. Jpn. (X-sen Bunseki no Shinpo, in Japanese), 2005, 36, 155.

20. http://www.amptek.com/.

21. "International Tables for X-ray Crystallography", ed. C. H MacGillavry and G. D. Rieck, 1962, Vol. III, Kynoch Press, Birmingham, 171. 